\title{
EMPODERAMIENTO Y AUTOESTIMA EN UN PRO- GRAMA DE ATENCIÓN PSICOSOCIAL DE VIOLENCIA
}

\section{Sofía Pereira Gatica' \\ Andrés Jiménez Figueroa ${ }^{2}$}

Resúmen: Se analiza las diferencias en los niveles de Empoderamiento, autoestima y violencia contra la mujer entre la etapa de ingreso y egreso en mujeres que asisten a un programa de atención psicosocial. A 142 mujeres se les administró la Escala de poder de decisión, la Escala de libertad de movimiento (CASTRO y CASIQUE, 2008), la Escala de Autoestima (ROSENBERG,1989) y la Pauta de Violencia Intrafamiliar (MINSAL, 2005) Se observan diferencias en el poder de decisión, la libertad de movimiento, autoestima e indices de violencia. Se concluye aumento del empoderamiento, autoestima y disminuye los índices de violencia, coherentes con la literatura revisada.

Palabras clave: Empoderamiento; Autoestima; Violencia.

Abstract: It analyzed the differences in levels of Empowerment, self-esteem and violence against women between the entry and exit stages in women attending a psychosocial care program are analyzed. 142 women were administered the Scale of decision-making power, the Scale of freedom of movement (CASTRO and CASIQUE, 2008), the Self-esteem Scale (ROSENBERG, 1989) and the Domestic Violence Pattern (MINSAL, 2005). Differences are observed in decision-making power, freedom of movement, self-esteem and violence rates. Increased empowerment, self-esteem and decreases violence rates, consistent with the literature reviewed.

Keywords: Empowerment; Self Esteem; Violence.

\section{Introducción}

La violencia contra la mujer es considerada, en distintos países, como uno de los problemas sociales más relevantes producto de las graves consecuencias que provoca en un gran número de mujeres en su vida personal y social (DOMÍNGUEZ, GARCIA y CUBEROS, 2008). Se constituye en un fenómeno universal, transmitido socialmente que ocurre sin distinción de nivel social, estudios, edad (BOSCH Y FERRER, 2005) e inclusive traspasa las barreras culturales,

\footnotetext{
1 Psicóloga, Universidad de Talca, E-mail: pereira.gatica@gmail.com

2 Psicólogo, Magíster en Administración y Dirección de Recursos Humanos, PhD. Administración de Empresas. Docente Investigador Universidad de Talca, Chile. E-mail: anjimenez@utalca.cl
} 
religiosas y sociales, ocupando en la actualidad prioridad dentro de los problemas de la salud pública. Se ha considerado que la violencia contra la mujer es en mayor medida transmitida a través de un proceso de socialización, donde hombres y mujeres aceptan la violencia justificándola, considerando su uso como parte del género masculino y naturalizando las conductas violentas (DUARTE, GÓMEZ y CARILLO, 2010).

La violencia resulta ser un problema relevante tanto en al ámbito económico como en el ámbito social en Latinoamérica (LODOÑO, GAVIRIA y GUERRE$R O, 2000$ ), evidenciada en diferentes situaciones sociales y manifestaciones, como por ejemplo: acoso físico moral en el trabajo, publicidad sexista, violencia derivada de los conflictos bélicos, agresiones físicas en la calle, tráfico de menores y otros (ALONSO y CASTELLANOS, 2006). La Organización Mundial de la Salud (2003) señala que la violencia es una de las principales causas de fallecimiento en el mundo para la población entre 15 y 44 años de edad, producto de las graves consecuencias ocasionadas hacia la integridad de las personas.

Desde una perspectiva legal, la violencia debe constituir un delito irrefutable sustentado en hechos concretos que ha generado graves consecuencias hacia otras personas. Por otra parte, la perspectiva de la salud pública, considera la violencia como aquella que ocasiona un daño físico, psicológico u otros que reduce la calidad de vida de las personas involucradas (HIIJAR, LOPPEZ y MUNOZ, 1997). La violencia de género ocurre tanto el ámbito privado como público, se trata de una violencia estructural que se dirige a las mujeres con el fin de mantener o aumentar su subordinación al género masculino hegemónico. Se sustenta a través de actitudes y creencias sexista (BOGANTES, 2008). La principal relevancia de la violencia de género es que causa más muertes que las enfermedades o los accidentes de tránsito (ACHARYA y STEVANATO, 2005), por lo que resulta importante elaborar estrategias o acciones para erradicarla.

Un sistema social basado en jerarquías, opresión y desigualdad constituye una de los factores de riesgo de la violencia contra la mujer (SAGOT, 2008). Además, de las creencias sobre los roles tradicionales respecto al hombre y la mujer posee relación con que los hombres ejerzan la violencia sobre la mujer y ellas aceptar tal comportamiento por parte de éstos (MULLENDER, 2000, YANES Y GONZÁLEZ, 2000, citado en SEBASTIÁN, et.al., 2010).

Los factores de riesgos identificados para las mujeres víctimas de violencia psicológica de acuerdo a la Encuesta de Victimización (2008) son mantener relaciones de tipo abusiva/controladora con la pareja, poseer creencias que justifican la agresión contra ellas y haber sido víctima de violencia física en la infancia (citado en SERVICIO NACIONAL DE LA MUJER, 2012). Las consecuencias que produce la violencia principalmente en la mujer, se pueden agrupar en tres grupos (SEPÚLVEDA, 2000, citado en AMOR, BOHÓRQUEZ Y ECHEBURUA, 2006). En primera instancia, son inmediatas correspondiente a las lesiones físicas 
- reacciones físicas como respuesta ante el estrés soportado (dolores de cabeza, problemas en el sistema inmunitario, etc.), posteriormente se encuentran las de largo plazo, que poseen un impacto más crónico como enfermedades médicas o psicosomáticas (trastornos psicológicos, secuelas anatómicas, funcionales, etc.), y el último grupo de consecuencias considera las consecuencias graves que pueden provocar la muerte de la mujer (episodios reiterados de maltrato, Femicidio).

La violencia posee como consecuencia para la mujer, en el ámbito físico: traumatismos, heridas, quemaduras, relaciones sexuales forzadas, enfermedades de transmisión sexual, embarazos de riesgos, abortos y la muerte. En el ámbito psicológico, la violencia produce trastornos por estrés post-traumático, ansiedad, depresión, intentos de suicidio, abuso del alcohol, las drogas y los psicofármacos, etc. Mientras que en el ámbito social, aislamiento, pérdida del empleo y absentismo laboral (FERNÁNDEZ, 2003).

Por su parte, autores como Aliaga, Ahumada y Marfull (2003, p.76) señalan que la violencia física contra las mujeres produce violencia psicológica como "problemas psicológicos, síndrome de estrés post traumático, miedo y ansiedad, sentimientos de vergüenza, inclusive el suicidio", en las consecuencias físicas se encuentran las "enfermedades ginecológicas y de transmisión sexual, dependencia a alcohol y sustancias, daños corporales, aborto, quejas somáticas, etc." Finalmente agregan consecuencias de índole sexual como disfunciones sexuales, fobias sexuales, entre otras.

En Chile, la violencia contra la mujer empezó a ser visualizada como un problema a través de la agenda pública en el año 1989 con la recuperación de la democracia y la creación del Servicio Nacional de la Mujer, donde las primeras acciones que tomó dicha institución fueron talleres regionales llamados "Talleres de resolución no violenta de conflictos: educación, cultura y cambio" (BUSTOS, 2001).

Actualmente, los programas están destinados a recibir mujeres de cualquier estatus social y profesional, que han sufrido o están sufriendo situaciones de ruptura con la pareja producto de la violencia (AMORÓS, et.al, 2007). Lo anterior, se debe a que en Chile a través de la agenda de género por parte el gobierno incorpora la perspectiva de género en las políticas públicas, estableciendo prioridades legislativas y de acción para erradicar la violencia contra las mujeres, por lo que debe asegurar que en todos los sectores estatales promuevan el derecho de las mujeres a una vida sin violencia, entreguen una oferta institucional que proteja a las víctimas de la violencia, repare el daño y que las empodere para el ejercicio de sus derechos (LARRAIIN, VALDEBENITO y RIOSECO, 2008). Un estudio realizado por Matud, Gutiérrez y Padilla (2004) se observo que los resultados del programa de intervención grupal indicaron que las mujeres presentaron una disminución significativa en su sintomatología como el estrés, depresión, ansiedad, aumentando su autoestima, confianza en sí misma y el control de sus vidas. 
En el año 1993, el Servicio Nacional de la Mujer realizó el primer estudio de prevalencia sobre la violencia intrafamiliar, evidenciando la violencia contra la mujer en larelación de pareja de un $59,7 \%$ siendo mayoritariamente mujeres casadas y principalmente violencia psicológica (SERVICIO NACIONAL DE LA MUJER, 2012). El Ministerio del Interior durante el año 2008 realizó una encuesta de victimización, la cual reflejó que el $35,7 \%$ de las mujeres reconoce haber sufrido violencia durante su vida. Además de señalar que 1 de cada 3 mujeres ha sido víctima de violencia alguna vez en su vida y que de las denuncias por violencia intrafamiliar el $85 \%$ son realizadas por mujeres (SERVICIO NACIONAL DE LA MUJER, 2012).

Las consecuencias sobre la violencia contra la mujer pueden ser fatales, debido a que las estadísticas obtenidas a través del Programa de Violencia Intrafamiliar de Chile Acoge, se observó que el número de femicidios ocurridos en el país durante el año 2011 fue de 40, a pesar de que existe una disminución de ellos en comparación al año 2008, donde se produjeron 59 femicidios, las cifras no dejan de ser preocupantes a nivel nacional (Servicio Nacional de la Mujer, 2011a). Por su parte, el Servicio Nacional de la Mujer (2011b) analizó durante el año 2010, la relación de la víctima-victimario de los femicidios ocurridos en ese año que fueron de 49 en total, de aquello el 34,7\% corresponde a cónyuges, $26,5 \%$ a convivientes, 18,4 ex convivientes, el $10,2 \%$ pololos y el 8,2 ex parejas/pololos.

Durante el año 2008, el Servicio Nacional de la Mujer realizó un estudio sobre la prevalencia de la violencia contra la mujer en la última década en Chile en al menos cuatro regiones: Región Metropolitana, Región de la Araucanía, Región de Coquimbo y la Región de Antofagasta. Los resultados señalaron un mayor porcentaje de violencia contra la mujer en la Región Metropolitana (RM) correspondiente al 50,3 seguida de la Región de Coquimbo 48,4\%. Además, se obtuvo en las cuatro regiones mayoritariamente violencia psicológica (RM 43,2\%, Araucanía 42,4\%, Coquimbo 41,9\% y Antofagasta 39,1\%) en comparación a la violencia física leve (RM 13,1\%, Araucanía 10,2\%, Coquimbo 13,4 y Antofagasta 11,1\%), (SERVICIO NACIONAL DE LA MUJER, 2008).

La presente investigación se enfoca principalmente en la violencia contra la mujer en las relaciones de pareja. En Chile, la ley de violencia intrafamiliar la estipula como todo maltrato que afecte la vida o integridad física o psíquica de quien tenga o haya tenido la calidad de cónyuge del ofensor o una relación de convivencia con él y la violencia de género como "toda conducta que atenta contra la dignidad e integridad física y moral de las mujeres, basada en la consideración social que tradicionalmente se les ha dado" (Instituto Andaluz de la Mujer, 2005, citado en Servicio Nacional de la Mujer, 2007, p. 80), con la finalidad de mantener o aumentar su subordinación al género masculino (GRACIA Y LILA , 2008). Por consiguiente, la violencia contra la mujer constituye una violación abierta a la Declaración Universal de los Derechos Humanos en cuanto a mermar 
la dignidad y derechos.

La violencia contra la mujer sitúa a un considerable número de mujeres a una situación de vulnerabilidad y aislamiento social, por lo que empoderarlas implicaría un cambio en la distribución de poder en las relaciones favoreciendo a que posean control en sus vidas (SAGOT, 2008), fortaleciendo la autoestima, la confianza en sí misma y la capacidad de elegir en su propia vida (CHARLIER, et. al., 2007). Se entenderá por empoderamiento al sentido de una mayor equidad de género, en el cual implica cambios en aspectos de la vida de las mujeres como autoestima, control sobre ingresos, acceso y control sobre recursos familiares, libertad de movimiento, participación en decisiones domésticas, capacidad de tomar decisiones sobre la crianza de los hijos, entre otros" (CASTRO y CASIQUE, 2008). Según Matud (2004) señala que aquellas mujeres que sufren violencia presentan síntomas somáticos, derivándose en depresiones, ansiedades, insomnio, lo que las llevaba a sentirse más inseguras, desvaloradas y poco apoyadas a diferencias de las mujeres que no experimentaban violencia.

La violencia contra la mujer que se produce de manera habitual y reiterada, puede provocar una disminución en su autoestima y sentimiento de autoeficacia (MATUD, GUTIÉRREZ y PADILLA, 2004). La autoestima se comprenderá como un sentimiento hacia uno mismo que puede ser negativo o positivo, el cual se construye por medio de una evaluación de las propias características (ROJAS-BARAHONA, ZEGERS Y FORSTER, 2009). La autoestima de una persona determina la capacidad para enfrentar las diversas situaciones de la vida cotidiana, donde una autoestima positiva favorece la satisfacción interna y la capacidad de superación frente a los problemas (CASIQUE y FUREGATO, 2007).

La presente investigación surge de la necesidad de determinar si existen diferencias significativas en las variables mencionadas en los programas actuales de intervención en el país. Bosch, Alzamora y Navarro (2006) consideran que los programas destinados a potenciar el empoderamiento en mujeres que sufren violencia, resultan ser importantes para combatir los sentimientos de inferioridad y culpa. Asimismo, la necesidad de establecer programas quepotencien el desarrollo personal, social y laboral, con la finalidad de fortalecer los factores de protección y disminuir los riesgos de sufrir violencia, promoviendo la autonomía (AMORÓS, et.al., 2007).

En Chile, no existen estudios enfocados en las variables de empoderamiento, autoestima y violencia contra la mujer, ni mucho menos en un contexto de atención psicosocial. Los programas de atención integral de violencia implementados por el Servicio Nacional de la Mujer, se han centrado en la atención física, psicológica y legal de las víctimas, pero han descuidado la capacitación de las mujeres para enfrentar su autonomía personal y económica (LARRAIIN, VALDEBENITO y RIOSECO, 2008). Se observa adicionalmente, que existe una falta de programas localizados en las zonas rurales, ya que estos generalmente 
se encuentran solo en capitales regiones, lo que resulta difícil la asistencia de los alrededores dada su conectividad (LARRAÍN, VALDEBENITO Y RIOSECO, 2008). Finalmente, se debe considerar que los programas de atención sobre la temática de la violencia, también provoca cierto desgaste profesional que puede llegar al agotamiento profesional o burnout, entre otras consecuencias.

El estudio del análisis del empoderamiento y violencia, se investigó en la Encuesta Nacional sobre la Dinámica de las relaciones en los hogares y Centro Regional de Investigaciones Multidisciplinarias ((UNAM), citado en Castro y Casique, 2008) en México. No obstante, en nuestro país aún no se realizan estudios en las variables empoderamiento, autoestima y violencia en su conjunto, ni menos en un contexto de atención enfocado en dichas variables.

La violencia hacia la mujer causa la pérdida de autoestima y capacidad de decisión (IBÁÑEZ, 2004, citado en MELGAR y VALLS, 2010). Lo cual se puede inferir que una baja autoestima provoca un estancamiento en la superación del problema, que en este caso de la violencia ejercida contra ella. Algunos estudios mencionan que la baja autoestima de las mujeres es lo que permite mantener la violencia del hombre hacia la mujer (FRANZOI, FONSECA y GUEDES, 2011).

La promoción de conductas y hábitos de vida saludables y la prevención de eventos dañitos para las comunidades y individuo priman en el quehacer de la salud pública, por lo que la intención preventiva es evitar que los hechos ocurran o se agraven sus consecuencias (CONCHA, 2002, p. 349). Por lo que los niveles de violencia dado su atención de prevención a que vuelva a ocurrir, deberían disminuir en comparación a su inicio.

En Chile, existe una institución que se dedica a entregar atención a las mujeres que son víctimas de violencia de pareja, catalogado como el Servicio Nacional de la Mujer (SERNAM) que opera a nivel regional a lo largo de todo el país. Dicha institución colabora en su gran mayoría a la superación de ésta temática a través de programas enfocados a las variables de estudio.

Es por esta razón, es imprescindible poder contar con los instrumentos validados y confiables para su utilización, desde ahí nace la importancia de validar la Escala de Diagnóstico de Violencia Intrafamiliar, ya que los instrumentos para la presente investigación son preestablecidos por el Servicio Nacional de la Mujery ocupados a nivel nacional para el programa de atención a sus víctimas.

Por otra parte, responder a la pregunta $¿$ Existen diferencias significativas en los índices de empoderamiento, autoestima en la violencia de pareja hacia la mujer según su etapa de ingreso y egreso en mujeres que sufren violencia de pareja y que asisten a un programa de atención psicosocial?, resulta fundamental dado que permitiría enfocar los aspectos principales para disminuir los niveles de violencia de pareja. Esto se debe a que dentro de los factores asociados con el riesgo de que un hombre maltrate a su pareja, indica que los factores individuales son 
depresión, poca instrucción, ingresos bajos, edad joven, entre otros, mientras que los factores de la relación el conflicto matrimonial, inestabilidad del matrimonio, etc. (OMS, 2003). Lo cual se puede inferir, que un bajo empoderamiento, referente a la poca instrucción y ingresos bajos, influiría en que la mujer reciba violencia. Esto concuerda con otros estudios, el cual indicaron que el $13 \%$ de las mujeres tuvieron que renunciar al trabajo remunerado a causa de la violencia contra ellas (OMS, 2003).

Algunos estudios señalan que los programas destinados a las mujeres con violencia de pareja, poseen como metas, aumentar la seguridad de la mujer, ayudarle a recuperar el control de su vida, remediar el impacto psicológico del abuso, en donde los resultados del programa de intervención grupal indicaron que las mujeres presentaron una disminución significativa en su sintomatología como el estrés, depresión, ansiedad, aumentando su autoestima, confianza en sí misma y el control de sus vidas (MATUD, GUTIÉRREZ y PADILLA, 2004).

\section{Método}

La población del estudio corresponde a 301 mujeres que sufren violencia en el ámbito de las relaciones de pareja en el contexto intrafamiliar, cuya edad sea mayor de 18 años y que residan, estudien o trabajen en alrededores de la ciudad de Curicó, ya que los Centros de la Mujer ubicados en comunas con población superior a 200.001 habitantes, deberán ingresar a 301 mujeres (SERVICIO NACIONAL DE LA MUJER, 2012). El muestreo utilizado fue de tipo no probabilístico intencionado y la muestra final corresponde a 142 mujeres que sufrieron violencia por su pareja y recibieron atención psicosocial, egresando de manera favorable del programa, cuyo rango de edad promedio corresponde entre los 26 y 65 años.

Para la evaluación de las variables de estudio se utilizaron como instrumentos de medición las fichas correspondientes a la etapa de ingreso y egreso, obtenidas a través del programa de atención psicosocial contenida por tres escalas:

1) Índice de Empoderamiento compuesta por la Escala de Poder de Decisión y Escala de Libertad de Movimiento o Autonomía (CASTRO Y CASIQUE, 2008). Estas escalas fueron analizadas en comparación a los resultados de la Encuesta Nacional sobre la Dinámica de las Relaciones en los Hogares (ENDIREH, 2006) y el Instituto Nacional de las Mujeres correspondiente al Centro regional de Investigación Multidisciplinarias (UNAM, 2008) en la ciudad de México.

2) Escala de Poder de Decisión comprende la octava sección en la Endireh (2006), el cual corresponde a la toma de decisiones en el hogar y la pareja, son medidas a través de 11 preguntas que permiten identificar quien decide la mayor parte de las veces en el hogar o en la relación de pareja. El modo de respuesta es 
solo él ( 1 punto), ambos ( 2 puntos), solo ella (3 puntos). El alfa de Cronbach es de 0,62 lo que indica que posee una consistencia interna.

3) Escala de Libertad de Movimiento o Autonomía comprende a la décima sección en la Endireh (2006), la cual corresponde a la autonomía de la mujer respecto a su esposo o pareja, son medidas a través de 7 preguntas y las alternativas de respuesta son: pedir permiso (1 punto), le avisa o solicita su opinión (2 puntos), no tiene que hacer nada (3 puntos), no va sola, va con él (0 puntos) y no lo hace ( 0 puntos). El alfa de Cronbach es e 0,73 lo que indica que posee una buena consistencia interna.

Escala de Autoestima de Rosenberg (1989). Consta de 10 ítems, puntuables entre 1 y 4 puntos, lo que permite obtener una puntuación mínima de 10 y máxima de 40 puntos. Las alternativas de respuesta son: muy de acuerdo (4 puntos), de acuerdo (3 puntos), en desacuerdo (2 puntos) y muy en desacuerdo (1 punto) (Rojas-Barahona, Zegers y Forster, 2009).La Escala de Autoestima ha sido validada en Chile, en el estudio de Rojas-Barahona y cols. (2009), en una muestra de jóvenes adultos, adultos y adultos mayores. La confiabilidad de la escala fue 0.754 , cumpliendo con los criterios de validez y confiabilidad para medir la autoestima en Chile, siendo similar a lo esperado por Rosenberg para la población original.

Pauta de Diagnóstico de Violencia Intrafamiliar de Ministerio de Salud (MINSAL, 2005) correspondiente a la guía clínica para la atención primaria de violencia intrafamiliar en la detección, diagnóstico y tratamiento, sección instrumentos de diagnóstico. El objetivo de la pauta es identificar el tipo de violencia presenta la mujer, ya sea psicológica, física, sexual y económica. El modo de respuesta es de tipo Likert donde la mujer debe responder una vez (1 punto), algunas veces ( 2 puntos), muchas veces ( 3 puntos) y no o nunca ( 0 puntos). El análisis de confiabilidad por consistencia interna fue de 0,88.

En relación a las dimensiones correspondientes a la violencia psicológica, física, sexual y económica, cuyos índices varían desde 0,62 para la dimensión de violencia económica hasta un 0,84 para la dimensión de violencia sexual.

La aplicación se realizó por los encargados del Centro de la Mujer de la Ciudad de Curicó, Chile a partir del mes de Julio del 2010 hasta Diciembre del año 2011. Los datos fueron analizados a través del programa estadísticos SPSS versión 15.0, para determinar si existen diferencias significativas en los índices de empoderamiento, autoestima y violencia contra la mujer en la relación de pareja, se utilizó comparación de medias (prueba t para muestras relacionadas).En segunda instancia, para identificar si existe relación entre las variables de estudio (empoderamiento, autoestima y violencia) y su dirección, se utilizó el estadístico coeficiente de correlación de Pearson. 
Tabla 1: Comparación de Medias en los índices de empoderamiento según la etapa de ingreso y egreso

\begin{tabular}{lcccccc}
\hline VARIABLES & M & DT & ETM & t & gl & Sig. \\
\hline Poder de Decisión Ingreso & 0,77 & 0,19 & 0,02 & $-9,986$ & 141 & $0,00^{*}$ \\
Poder de Decisión Egreso & 0,94 & 0,11 & 0,01 & & & \\
Libertad de Movimiento Ingreso & 0,64 & 0,30 & 0,03 & $-9,001$ & 141 & $0,00 *$ \\
Libertad de Movimiento Egreso & 0,90 & 0,20 & 0,02 & & & \\
\hline
\end{tabular}

* $p<0,001$

Fuente: Elaboración Propia (2019).

En la tabla 2 los resultados muestran diferencias significativas $(t=-0,751 ; g l=$ 141; $p<0,001)$ en la autoestima, donde en la etapa de egreso las mujeres $(M=29,85)$ presentan una mayor puntuación de la autoestima que en la etapa de ingreso.

Tabla 2: Comparación de Medias en los índices de autoestima según la etapa de ingreso y egreso

\begin{tabular}{lcccccc}
\hline VARIABLES & M & DT & ETM & t & gl & Sig. \\
\hline Autoestima Ingreso & 25,56 & 4,81 & 0,40 & $-0,751$ & 141 & $0,00 *$ \\
Autoestima Egreso & 29,85 & 4,43 & 0,37 & & & \\
\hline${ }^{*} p<0,001$ & & & & & & \\
\hline
\end{tabular}

Fuente: Elaboración Propia (2019).

En la tabla 3, se observó diferencias significativas ( $t=20,701 ; g l=141 ; p<0,001$ ) en los índices de violencia psicológica, donde en la etapa de ingreso las mujeres $(M=20,75)$ presentan un mayor índice de violencia que en la etapa de egreso; diferencias significativas $(t=12,696 ; g l=141 ; p<0,001)$ en los índices de violencia física, donde en la etapa de ingreso las mujeres $(M=5,68)$ presentan un mayor índice de violencia física que en la etapa de egreso; diferencias significativas ( $t=10,537 ; g l=141 ; p<0,001$ ) en los indices de violencia sexual, donde en la etapa de ingreso las mujeres $(M=4,07)$ presentan un mayor índice de violencia sexual que en la etapa de egreso y diferencias significativas $(t=9,673 ; g l=141 ; p<0,001)$ en los índices de violencia económica, donde en la etapa de ingreso las mujeres $(M=3,94)$ presentan un mayor índice de violencia económica que en la etapa de egreso. 
Tabla 3: Comparación de Medias en los índices de violencia contra la mujer en la relación de pareja según la etapa de ingreso y egreso

\begin{tabular}{lcccccc}
\hline VARIABLES & M & DT & ETM & t & gl & Sig. \\
\hline Violencia Psicológica Ingreso & 20,75 & 7,32 & 0,61 & 20,701 & 141 & 0,00 * \\
Violencia Piscológica Egreso & 5,71 & 4,83 & 0,41 & & & \\
Violencia Fisica Ingreso & 5,68 & 4,83 & 0,41 & 12,696 & 141 & 0,00 * \\
Violencia Fisica Egreso & 0,21 & 1,36 & 0,11 & & & \\
Violencia Sexual Ingreso & 4,08 & 4,23 & 0,36 & 10,537 & 141 & $0,00 *$ \\
Violencia Sexual Egreso & 0,23 & 1,22 & 0,10 & & & \\
Violencia Económica Ingreso & 3,94 & 3,61 & 0,30 & $-9,673$ & 141 & 0,00 * \\
Violencia Ecónomica Egreso & 0,52 & 1,76 & 0,15 & & & \\
\hline${ }^{*} p<0,001$ & & & & & & \\
\hline
\end{tabular}

Fuente: Elaboración Propia (2019).

En la tabla 4, se presentan las correlaciones entre el poder de decisión y los tipos de violencia, donde se observa que el poder de decisión se relaciona significativamente con $(r=-0,561 ; p<0,01)$ la violencia psicológica, con $(r=-0,302$; $p<0,01)$ la violencia física, con $(r=-0,407 ; p<0,01)$ la violencia sexual y $(r=$ -0,335; $p<0,01)$ la violencia económica. Específicamente, se observa correlaciones de magnitud media alta y negativa con la violencia psicológica y correlaciones de magnitudes medias bajas y negativas con la violencia física, sexual y económica.

Tabla 4: Relaciones entre Poder de Decisión y los tipos de violencia contra la mujer en la relación de pareja

\begin{tabular}{llcc}
\hline VARIABLES & & \multicolumn{2}{c}{ Correlación sig. (bilateral } \\
\hline Libertad de Movimiento & Violencia Psicológica & $-0,526$ & ${ }^{* *}$ \\
\hline & Violencia Fisica & $-0,264$ & ${ }^{* *}$ \\
\cline { 2 - 4 } & Violencia Sexual & $-0,312$ & ${ }^{* *}$ \\
\cline { 2 - 4 } & Violencia Económica & $-0,272$ & ${ }^{* *}$ \\
\hline & ${ }^{*} \mathrm{p}<0,05 ;{ }^{* *} \mathrm{p}<0,01 ;{ }^{* * *} \mathrm{p}<0,001$ & \\
\hline
\end{tabular}

Fuente: Elaboración Propia (2019).

En la tabla 5, se presentan las correlaciones entre libertad de movimiento y los tipos de violencia, donde se observa que la libertad de movimiento se relaciona significativamente con $(r=-0,526 ; p<0,01)$ la violencia psicológica, con $(r=$ $-0,0264 ; p<0,01)$ la violencia física, con $(r=-0,312 ; p<0,01)$ la violencia sexual y $(r=-0,272 ; p<0,01)$ la violencia económica. Específicamente, se observa correlaciones de magnitud media alta y negativa con la violencia psicológica y correlaciones de magnitudes medias bajas y negativas con la violencia física, sexual y económica. 
Tabla 5: Relaciones entre Libertad de Movimiento y los tipos de violencia contra la mujer en la relación de pareja

\begin{tabular}{llcc}
\hline VARIABLES & & \multicolumn{2}{c}{ Correlación sig. (bilateral } \\
\hline Libertad de Movimiento & Violencia Psicológica & $-0,526$ & ${ }^{* *}$ \\
\hline & Violencia Física & $-0,264$ & ${ }^{* *}$ \\
\cline { 2 - 4 } & Violencia Sexual & $-0,312$ & ${ }^{* *}$ \\
\cline { 2 - 4 } & Violencia Económica & $-0,272$ & ${ }^{* *}$ \\
\hline & ${ }^{*} \mathrm{p}<0,05 ;{ }^{* *} \mathrm{p}<0,01 ;{ }^{* * *} \mathrm{p}<0,001$ & \\
\hline
\end{tabular}

Fuente: Elaboración Propia (2019).

Respecto a la relación entre el poder de decisión y la autoestima, donde se observa que el poder de decisión se relaciona significativamente con la autoestima, específicamente se observa una correlación baja y positiva $(r=0,311 ; p<0,01)$. Entre libertad de movimiento y autoestima, se observa que la libertad de movimiento se relaciona significativamente con la autoestima, específicamente se observa una correlación baja y positiva $(r=0,41 ; p<0,01)$ con la autoestima.

En la tabla 6 , se presentan las correlaciones entre la autoestima y los tipos de violencia, donde se observa que la autoestima se relaciona significativamente con $(r=-0,276 ; p<0,01)$ la violencia psicológica y la violencia sexual $(r=-0,181$; $p<0,01)$. Específicamente, se observa correlaciones de magnitudes bajas y negativas con la violencia psicológica y sexual.

Tabla 6: Relaciones entre Libertad de Movimiento y los tipos de violencia contra la mujer en la relación de pareja

\begin{tabular}{llcc}
\hline VARIABLES & & \\
\hline Autoestima & & Correlación & Sig. \\
\hline & Violencia Psicológica & $-0,276$ & $* *$ \\
\cline { 2 - 4 } & Violencia Física & $-0,112$ & \\
\cline { 2 - 4 } & Violencia Sexual & $-0,181$ & $*$ \\
\cline { 2 - 4 } & Violencia Económica & $-0,036$ & \\
\hline & $*$ p $<0,05 ;{ }^{* *} \mathrm{p}<0,01 ;{ }^{* * *} \mathrm{p}<0,001$ \\
\hline
\end{tabular}

Fuente: Elaboración Propia (2019).

\section{Conclusiones}

Los resultados obtenidos en la presente investigación, se concluye que el objetivo principal correspondiente a determinar diferencias significativas entre los indices de empoderamiento, autoestima y violencia contra la mujer en la relación de pareja según la etapa de ingreso y egreso en mujeres que asisten a un programa de atención psicosocial, fue respondido de forma satisfactoria confirmándose todas las hipótesis planteadas. 
La primera hipótesis señala la existencia de diferencias significativas en los indices de empoderamiento según la etapa de ingreso y egreso, es confirmada, encontrándose que en la etapa de egreso las mujeres obtuvieron una mayor puntuación de poder de decisión y libertad de movimiento que en la etapa de ingreso. Esto coincide con los hallazgos expuestos por Matud, Gutiérrez y Padilla (2004), quienes indican que aquellas mujeres en situación de violencia al finalizar un programa de intervención enfocado a aumentar su seguridad y control en sus vidas, presentaron una disminución significativa en su sintomatología y aumentando su autoestima y control en sus vidas. Se complementa con lo demostrado por Sagot (2008) quien encontró que la violencia produce desempoderamiento y exclusión social en las mujeres, siendo el empoderamiento un cambio en la distribución del poder en las relaciones, favoreciendo a las mujeres que sufren violencia obtengan un mayor control en sus vidas.

Los roles rígidos y conservadores atribuidos al hombre y a la mujer, se relaciona con la violencia que ejerce al hombre hacia la mujer (GONZALEZ, ECHEBURÚA y CORRAL, 2008), por lo que la posesión y control de los recursos, el trabajo y los productos están asociados a la masculinidad (DAVICO, 2004), por lo que concuerda que lo esperado que los índices de empoderamientos fueran bajos en su ingreso.

La segunda hipótesis señala la existencia de diferencias significativas en los índices de autoestima según la etapa de ingreso y egreso, es confirmada, encontrándose que en la etapa de egreso las mujeres obtuvieron una mayor puntuación de autoestima que en la etapa de ingreso. Esto coincide con lo encontrado por Matud, Gutiérrez y Padilla (2004), quienes señalan que la violencia contra la mujer cuando se produce de forma reiterada, provoca una disminución en la autoestima y el sentimiento de autoeficacia y se complementa con lo expuesto por Franzoi, Fonseca y Guedes (2011), quienes indican que una disminuida autoestima en las mujeres es lo que permite mantener la violencia del hombre hacia la mujer, ya que la vincula a un género débil y con dificultad para resolver los conflictos.

Por su parte, Matud (2004) encontró que las mujeres que poseen una mayor confianza en sí mismas y que tienen una valoración positiva, indican tener una mejor salud, evidenciado en menos síntomas depresivos, de ansiedad y somáticos, debido a que poseen un estilo de afrontamiento más racional de aquellas mujeres que poseen una desvalorización de sí mismas.

La tercera hipótesis señala la existencia de diferencias significativas en los índices de violencia contra la mujer en la relación de pareja según la etapa de ingreso y egreso, en donde las mujeres presentaron una mayor puntación de violencia en la etapa de ingreso que en su egreso, si confirma lo esperado por Duarte, Gómez y Carillo (2010), quienes mencionan que hombres y mujeres aceptan la violencia a través de justificaciones y naturalidad, destacándo que las conductas 
violentas al interior de la pareja, tanto hombres como mujeres lo consideran una forma válida para resolver los conflictos.

A su vez, se complementa con lo expuesto por Aliaga, Ahumada y Marfull (2003) quienes mencionan que la violencia posee consecuencias psicológicas, fisicas y sexuales. Al observar de forma detallada, se evidencia que las diferencias encontradas, la violencia psicológica posee los mayores índices que los otros tipos de violencia en la etapa de ingreso. Esto concuerda con lo mencionado por Sarasusa, et.al. (2007), quienes indican que la severidad de la violencia depende de las consecuencias y la frecuencia/intensidad del maltrato, pero no necesariamente por la violencia física ejercida. Lo cual se agrega algunos estudios, que evidencian que las mujeres en situación de violencia pueden tardar alrededor de 10 años en promedio en solicitar ayuda (LABRADOR, FERNÁNDEZ y RINCÓN, 2006). Por su parte, el Servicio Nacional de la Mujer en el año 2008, realizó un estudio en cuatro regiones del país, donde los resultados demostraron que las mujeres sufrían principalmente violencia psicológica en comparación a la violencia física. Lo anterior, se agrega que en la provincia de Curicó durante el año 2010, el mayor número de denuncias por parte de las mujeres por lesiones psicológicas (1050) en comparación a las leves (384).

Por el contrario, las diferencias significativas encontradas en cuanto a una mayores índices de violencia en la etapa de ingreso que en su egreso, concuerda con lo expuesto por Concha (2002) que menciona que la promoción de conductas y hábitos de vida saludables y la prevención de eventos dañinos para la comunidad y el individuo priman en el accionar de la salud pública, por lo que la intención preventiva es evitar que los hechos ocurran o que se agraven las consecuencias.

En relación a la cuarta hipótesis, que señala que existe una relación entre el empoderamiento y la violencia contra la mujer en la relación de pareja entre la etapa de ingreso y egreso, es confirmada. Esto concuerda con lo expresado por GUTIERREZ ET. AL (2010), quienes mencionan que cuando es más frecuente el intento de abandono de la situación de violencia, es cuando tienen autonomía económica o en la esfera pública, el maltrato es severo, si cuentan con apoyo familiar y social y si el maltrato afecta directamente a sus hijas e hijos, ya que dentro de los factores que pueden influir en que las mujeres permanezcan en una relación de violencia, es en base a una dependencia económica y afectiva (FRANZOI, FONSECA y GUEDES, 2011).

Además, el empoderamiento genera cambios en aspectos de la vida de las mujeres como "autoestima, control sobre ingresos, acceso y control sobre recursos familiares, libertad de movimiento, participación en decisiones domésticas, capacidad de tomar decisiones sobre la crianza de los hijos, control sobre las relaciones sexuales, ausencia de la violencia, conocimiento en temas legales, entre otros" (MALHOTRA y COLS., 2002, citado en CASTRO y CASIQUE, 2008, 
p. 45), lo cual éstos cambios provocarían una disminución en la violencia al otorgarle a la mujer igualdad de condiciones, recuperando su dignidad como persona y pueda tomar sus propias decisiones (BOSH, et.al, 2005).

La quinta hipótesis sobre si existe una relación entre el empoderamiento y autoestima de la mujer entre la etapa de ingreso y egreso, resulta ser comprobada con lo hallado por Charlier, et.al. (2007), quienes mencionan que el empoderamiento está relacionado con la toma de poder, fortaleciendo la autoestima, la confianza en sí misma y la capacidad de elegir en su propia vida. Autores como Bosch, Alzamora y Navarro (2006) consideran que los programas destinados a potenciar el empoderamiento en mujeres que sufren violencia, resultan ser importantes para combatir los sentimientos de inferioridad, culpa, entre otros.

La sexta hipótesis señala la existencia de una relación entre la autoestima de la mujer y la violencia contra la mujer en la relación de pareja, es confirmada, ya que los resultados son coincidentes con los hallazgos que encontraron que entre menor sea la autoestima es mayor la probabilidad de padecer violencia dentro de una relación de pareja, debido a que la autoestima regula procesos interpersonales significativos, dentro de estos se incluyen desde el cómo se perciben los afectos, las motivaciones y la información, hasta la elección de pareja (ADMAS y CERVANTES, 2012). Por su parte, GONZALEZ Y ROJAS (2000) señalan que la autoestima negativa se va forjando cuando no hay espacios para la crítica constructiva ni la autonomía; cuando no se reciben expresiones de afecto y apoyo y cuando las posibilidades de desarrollo se ven restringidas por su entorno, como sería en el caso de las mujeres que son violentadas por sus parejas.

La presente investigación al utilizarse instrumentos cuantitativos posee la ventaja de medir de forma cuantificable las variables en estudio, lo que permite obtener registros comparables entorno a una situación, sin embargo, posee la limitación de no contemplar las creencias o percepciones sobre la violencia o estilos de crianza por parte de las mujeres. Por otro lado, al ser instrumentos preestablecidos no fue posible incluir o medir otras variables que podrían ser relevantes como la percepción que poseen los hombres en torno al tema. Otra de las limitaciones, es que los instrumentos para medir el empoderamiento resultaron incorporarse recientemente en los programas del Servicio Nacional de la mujer, ya que los programas de atención integral de violencia implementados por dicha institución, se han centrado en la atención física, psicológica y legal de las víctimas, pero han descuidado la capacitación de las mujeres para enfrentar su autonomía personal y económica (LARRAíN, VALDEBENITO y RIOSECO, 2008), junto con no haber sido estudiadas en su conjunto por nuestro país.

Respecto a la muestra, resulta como limitante enfocarse solamente en aquellas mujeres que han tenido o tienen una relación de convivencia o de cónyuges, ya que la ley no contempla otras relaciones en la pareja. Es así, como se recomienda realizar estudios enfocados a las relaciones que ocurren en el pololeo, las relacio- 
nes de homosexuales, entre otras, con la finalidad de elaborar políticas públicas dirigidas a este tipo de población.

Finalmente, se debería incorporar variables como prevalencia de retractación de las denuncias, etnias, como es percibida la violencia en contextos rurales, etc. En cuanto a las políticas públicas, deberían enfocarse en obtener un registro único y realizar seguimientos personalizados a las víctimas.

\section{Referencias}

ACHARYA, A. Y STEVENATO, A. (2005). Violencia y tráfico de mujeres en México: una perspectiva de género. Revistas Estudos Feministas, 13 (3), 507524.

ADAMS, J.; CERVANTES, L.. Violencia pasiva en mujeres universitarias: Un estudio exploratorio de las causas del deterioro de la autoestima. Psicología y Salud; 2012, n²2 (1), p. 133-139.

ALIAGA P.; AHUMADA, G.; MARFULL, M.. Violencia hacia la mujer: un problema de todos. Revista Chilena de obstetricia y ginecología: 2003, $n^{0} 68,(1)$, p. 75-78.

ALONSO, J. Y CASTELLANOS, J. (2006). Por un enfoque integral de la violencia intrafamiliar. Intervención Psicosocial, 15 (3), 253-274.

AMOR, P., BOHÓRQUEZ, A. Y ECHEBURÚA, E. (2006). ¿Por qué y a qué coste físico y psicológico permanece la mujer junto a su pareja maltratadora?. Acción Psicológica, 4 (2), 129-154.

AMORÓS, P.; RODRIGO, M. J.; DONOSO, T; MÁIQUEZ, M. L.. Diseño de materiales para la formación en género. El programa de apoyo psicosocial para mujeres. Revista Fuentes: $2007, n^{\circ} 7$, p. 37-51.

BOGANTES, J. (2008). Violencia Doméstica. Medicina Legal de Costa Rica, 25 (2), 55-60.

BOSCH, E.; FERRER, V.; ALZAMORA, A.; NAVARRO, C.. Itinerarios hacia libertad: La recuperación integral de las víctimas de la violencia de género. Psicología y Salud: 200515 (1), 97-105.

BUSTOS, C. (2001). Políticas Públicas para la Prevención de la Violencia Intrafamiliar: Análisis del Programa de Resolución no violenta de conflictos, Sernam, VIII Región. Extraído el 22 de enero del 2012 desde http://www.trabajosocialudec.cl/rets/wp-content/uploads/2011/02/POL\%C3\%8DTICAS-P\%C3\%9ABLICAS-PARA-LA-PREVENCI\%C3\%93N-DE-LA-VIOLENCIA- 
-INTRAFAMILIAR.pdf

CASIQUE, I.; FERREIRA, A.. Violenceagainstwomen: Theoretical reflections. Revista Latinoamericana de Enfermería: 2007, 14 (6), 116-123.

CASIQUE, L.; FUREGATO, A.. Violencia perpetrada por compañeros íntimos en mujeres. Index de Enfermería: 2007, 16 (56), 17-21.

CASTRO, R.; CASIQUE, I.. Análisis de resultados de la Encuesta Nacional sobre la Dinámica de las Relaciones en los Hogares 2006. 2008. Extraído el 12 de enero del 2012 desde http://cedoc.inmujeres.gob.mx/documentos_download/100925.pdf.

CHARLIER, S., CAUBERGS, L., MALPAS, N. Y MULA, E.. El Proceso de empoderamiento de las mujeres. Guía Metodológica.2007, extraído el 10 de diciembre del 2011 desde http://www.atol.be/docs/publ/gender/proceso_empoderamiento_mujeres_CFD.pdf.

CONCHA, A.. Impacto social y económico de la violencia en las Américas. Revista del Instituto Nacional de Salud: 2002, 22 (2), 347-361.

DAVICO, G. (2004). Empoderamiento femenino a través de programas de microcrédito en Argentina. Extraído el 10 de enero del 2012 desde http://www. salvador.edu.ar/csoc/idicso/docs/ponenciadavico.pdf

DOMÍNGUEZ, J.M.; GARCÍA, P.; CUBEROS I.. Violencia contra las mujeres en el ámbito doméstico: consecuencias sobre la salud psicosocial. Anales de Psicología: 2008, 24 (1), 115-120.

DUARTE, E.; GÓMEZ, J.; CARILLO, C.. Masculinidad y hombre maltratador ¿Pueden las creencias e hombres y mujeres propiciar violencia de género? Revista de Psicología: 2010, 19 (2), 7-30.

FERNÁNDEZ, C. (2003). Salud Pública: Violencia Doméstica. Extraído el 19 de Noviembre del 2011 desde http://www.msps.es/ciudadanos/violencia/docs/ VIOLENCIA_DOMESTICA.pdf

FRANZOI, N.; GODOY, R.; NUNES, R.. Violencia de género: concepciones de profesionales de los equipos de salud de la familia. Revista Latinoamericana de Enfermería: 2011, 19(3), 589-597.

GRACIA, E.; LILA, M.. Los profesionales de la salud y la prevención de la violencia doméstica contra la mujer. Revista médica de Chile: 2008, 136 (3), 394400.

GONZÁLEZ-ORTEGA, I., ECHEBURÚA, E. Y DE CORRAL, P. (2008). Va- 
riables significativas en las relaciones violentas en parejas jóvenes: una revisión. Psicología Conductual, 16 (2), 207-225.

GONZALEZ, P. y ROJAS, G. (2000). Violencia conyugal, trabajo y salud mental, desde una perspectiva psicosocial: estudio exploratorio descriptivo del bienestar psicosocial en mujeres que viven violencia conyugal en las comunas de Valparaíso y Viña del mar, en relación a su situación laboral. Extraído el 22 de Diciembre del 2011 desde http://revistas.ojs.es/index.php/index/index

GUTIÉRREZ, A., ARMIENTA, L. JAIME, S. Y MAYORAL, M. (2010). Modelo de Prevención de la Violencia de Género. Extraído el 4 de marzo del 2012 desde http://cedoc.inmujeres.gob.mx/ftpg/Sonora/sonmeta5_1.pdf

HÍJAR, M., LÓPEZ, M. Y MUÑOZ, J. (1997). La violencia y sus repercusiones en la Salud: Reflexiones Teóricas y Magnitud del Problema en México. Salud Pública de México, 29 (6), 1-8.

LABRADOR, F.; FERNÁNDEZ, M.; RINCÓN, P.. Eficacia de un programa de intervención individual y breve para el trastorno por estrés postraumático en mujeres victimas de violencia doméstica. International Journal of Clinical and Health Psychology: 2006, 6 (3), 527-547.

LARRAIIN, S.; VALDEBENITO, L.; RIOSECO, L.. La situación de violencia contra las mujeres en Chile. Legislación y Políticas Públicas. 2008, extraído el 12 de diciembre del 2011 desde http://portal.unesco.org/geography/es/files/11343/12436345861Resumen_ejecutivo_del_estudio_la_situacion_de_violencia_contra_las_mujeres_en_Chile._Legislacion_y_politicas_publicas.pdf.

LODOÑO, J., GAVIRIA, A., Y GUERRERO, R. (2000). Asalto al Desarrollo: Violencia en América Latina. Banco Interamericano de Desarrollo. Extraído el 1 de Marzo del 2012 desde http://books.google.es/books?hl=es\&lr=\&id=xbtm$\mathrm{dBLGmFOC \& oi=fnd \& pg=PA11}$

MATUD, M.. Autoestima en la Mujer: Un análisis de su relevancia en la Salud. Avances en Psicología Latinoamericana: 2004, 22, 129.140.

MATUD, M., GUTIÉRREZ, A. Y PADILLA, V.. Intervención Psicológica con mujeres maltratadas por su pareja. Papeles del Psicólogo: 2004, 25 (88), 1-9.

MELGAR, P. Y VALLS, R. (2010). Estar enamorada de la persona que me maltrata: socialización en las relaciones afectivas y sexuales de las mujeres víctimas de violencia de género. Trabajo Social Global, 1 (2), 149-161.

MINISTERIO DE SALUD - MINSAL. Guía Clínica para la Atención Primaria. Detección, Diagnóstico y Tratamiento de Violencia Intrafamiliar. MINSAL, Gobierno de Chile: 2005. 
ORGANIZACIÓN MUNDIAL DE LA SALUD - OMS. Informe Mundial sobre la violencia y la salud. OMS: 2003. Extraído el 12 de febrero del 2012 desde http://mayores.pre.cti.csic.es/documentos/documentos/oms-informeviolencia-01.pdf.

ROJAS-BARAHONA, C.; ZEGERS, B.; FORSTER, C.. La escala de autoestima de Rosenberg: Validación para Chile en una muestra de jóvenes adultos, adultos y adultos mayores. Revista Médica, Chile: 2009, 137 (6), 791-800.

ROSENBERG, M. (1989). Society and the adolescent Self-image. Revised edition. Middle-town, CT: Wesleyan University Press.

SAGOT, M.. Los límites de las reformas: violencia contra las mujeres y políticas públicas en América Latina. Revista de Ciencias Sociales: 2008, 2 (120), 35-48.

SARASUSA, B.; ZUBIZARRETA, I.; Echeburúa, E.; Corral, P.. Perfil psicopatológico diferencial de las víctimas de violencia de pareja en función de la edad. Psicothema: 2007, 19 (3), 459-466.

SEBASTIÁN, J., ORTIZ, B., GIL, M., GUTIÉRREZ DEL ARROYO, M., HERNÁINZ, A. Y HERNÁNDEZ, J. (2010). Violencia en las parejas jóvenes: ¿Cuál es el camino a seguir?. Clínica Contemporánea, 1 (2), 71-83.

SERVICIO NACIONAL DE LA MUJER, SERNAM (2011a). Estatísticas relacionadas: Número de femicidios íntimos ocurridos en Chile entre 2007 y julio 2011. Extraído el 25 de enero del 2012 desde http://estudios.sernam.cl/?m=s.

SERVICIO NACIONAL DE LA MUJER, SERNAM (2011b). Porcentaje de Femicidios íntimos 2010 según víctima-victimario. Extraído el 24 de agosto de 2011, desde http://estudios.sernam.cl/?m=s\&i=35

SERVICIO NACIONAL DE LA MUJER, SERNAM (2012). Violencia Intrafamiliar. Extraído el 2 de marzo del 2012 desde http://estudios.sernam.cl/?m=t\&i=6

Recebido em agosto de 2018.

Aprovado em dezembro de 2018. 